\title{
Older Adults with COVID-19 Can Choose Care at Home: Lessons Learned from New York City
}

\author{
Justina L. Groeger, MD, MPH ${ }^{1,2}$ (D) Joanna L. Starrels, MD, $M S^{1,2}$, and Amy R. Ehrlich, MD ${ }^{3,4}$
}

'Division of General Internal Medicine, Montefiore Health SystemBronx, NY, USA; ${ }^{2}$ Division of General Internal Medicine, Albert Einstein College of Medicine Bronx, NY, USA; ${ }^{3}$ Division of Geriatrics, Montefiore Health SystemBronx, NY, USA; ${ }^{4}$ Division of Geriatrics, Albert Einstein College of MedicineBronx, NY, USA.

J Gen Intern Med 35(9):2755-6

DOI: $10.1007 / \mathrm{s} 11606-020-06035-2$

(c) Society of General Internal Medicine 2020

I nternists often have conversations about goals of care for patients at the end-of-life. Discussions are usually informed by hospital data or a witnessed functional decline over time for patients at home. However, COVID-19 is not usual. Providing ambulatory and inpatient care in the epicenter of New York City (NYC), we have witnessed many frail, older adults acutely decline due to COVID-19. Because hospitals limit or deny visitors to COVID-19 patients, many older adults fear dying there alone and wish to remain at home with their family. Unfortunately, the presentation of COVID-19 often includes rapid onset of delirium, so we miss having these conversations in advance.

In April, before the pandemic peaked in NYC, the family of a patient asked us for help treating him at home. They suspected that a urinary tract infection was causing his fever and lethargy. We discussed the risks and benefits of treating him empirically during the global pandemic since he was too weak to leave home for testing. And, if he did not already have COVID-19, the family did not want to risk exposing him. Antibiotics did not help; he became more lethargic and suddenly developed respiratory distress. Despite hoping to avoid hospitalization, they brought him to the Emergency Department (ED) where he tested COVID-19 positive. We had already discussed his goals of care; they did not want intubation or cardiopulmonary resuscitation (CPR). He died comfortably in the ED, receiving high-flow nasal cannula and morphine for air hunger. In his final moments, his family did not agonize over these decisions. Acute respiratory distress can be difficult and scary to manage at home, often resulting in an ED visit for patients who intended to stay home. Even when this

Received June 19, 2020

Accepted July 1, 2020

Published online July 14, 2020 occurs, clarifying goals of care beforehand with family and ED staff can ensure that goals are met.

For frail older adults, whether to hospitalize is no simple question, and the pandemic further complicates it. Right now, hospitals are disorienting places for anyone. Personal protective equipment (PPE) renders nurses and physicians unidentifiable. Hospital staff limit time with patients to reduce their own COVID-19 exposure and to preserve PPE. Without family helping them, older adults easily become delirious in this unfamiliar setting. Home is a familiar place where they can receive 24/7 attention, love, and comfort from their family. Despite hospital support like increased monitoring, intravenous medications, hemodialysis, high-flow oxygen, and intubation, up to $27 \%$ of patients 85 and older and $11 \%$ of patients 65-84 die from COVID-19 infection. ${ }^{1-3}$ Old age is a predictor of dementia and severe COVID-19 disease, and adults with dementia are more likely to have comorbidities (diabetes, cardiovascular disease, and pneumonia) associated with poor outcomes, including mortality, from COVID-19 infection. ${ }^{4}$

So we must be proactive. For patients at risk of severe disease due to COVID-19, we now frankly discuss the benefits and risks of hospitalization with patients and caregivers before patients become ill. During these difficult conversations, we are frequently covering for colleagues deployed in the hospital or ill themselves, and often have not met the patient beforehand. Most of the data we base our guidance on is from a single phone conversation with the patient and caregivers. They identify a health care proxy or next of kin, and we learn the patient's wishes about seeking emergency care, hospitalization, and if necessary, dialysis, intubation, and resuscitation. Determining goals of care and code status beforehand makes hospital care smoother and better aligned with patients' wishes - which we know only when we ask. Some patients will choose hospital admission, and others to stay home or return home after an ED visit for COVID-19 symptoms. But for those who avoid hospitalization, the decision should be thoughtful, not made from fear.

For patients who wish to remain at home, but who may later consider hospitalization, Certified Home Health Agencies (CHHAs) provide skilled home nursing and rehabilitation even during the pandemic. Despite initial scaling back of home care in NYC due to inadequate PPE, we have facilitated home visits for symptom management, vital signs and pulse 
oximetry readings, phlebotomy, and physical therapy for deconditioning. We can also provide home oxygen. Following the national emergency declaration, the Centers for Medicare and Medicaid (CMS) liberalized the regulations that would have denied home COVID-19 patients easy access to oxygen. ${ }^{5,6}$ CHHAs can help coordinate the delivery of hospital beds and other medical equipment to meet patients' functional needs at home.

For patients with high risk of mortality who prefer palliation of symptoms, hospice programs can provide many in-home services, including nursing care, 24/7 phone support, medical supplies and equipment, pastoral care, short-term inpatient hospice care, and respite care. Given the poor prognosis for many with severe COVID-19, hospice programs in NYC began interpreting admissions criteria to include frail patients with acute functional decline due to COVID-19. This can be applied nationwide. One patient with mild cognitive impairment and stable pulmonary disease was not a hospice candidate before the pandemic. But she became eligible when we diagnosed her by telephone with acute functional decline from suspected COVID-19 after she was newly bedbound and not eating or communicating clearly. Her daughter said her mother wished to avoid hospitalization and aggressive care. We referred her to hospice care, at home, and she remains with her family.

Primary care providers can and should prevent hospitalization for frail older patients who have an acute decline due to COVID-19 and prefer the comfort of home with their loved ones. In our experience with older COVID-19 adults, declines occur suddenly and delirium is common, even absent a history of cognitive impairment. So proactive conversations are essential. For providers who otherwise feel powerless against COVID-19, it is reassuring to align the care plan with a patient's wishes. As the pandemic alters how we deliver care, we call on all primary care providers to identify patients at high risk of mortality from COVID-19 and speak candidly with them, while we still can. ${ }^{7}$
Corresponding Author: Justina L. Groeger, MD, MPH; Division of General Internal Medicine, Albert Einstein College of Medicine Bronx, NY, USA (e-mail: jugroege@montefiore.org).

Funding Information AHRQ K12HSO26396 (Groeger), NIDA R01DA039046 (Starrels), NIDA K24DA046309 (Starrels).

\section{Compliance with Ethical Standards:}

Conflict of Interest: The authors have no conflicts of interest to declare for this article.

\section{REFERENCES}

1. CDC Covid- Response Team. Severe outcomes among patients with coronavirus disease 2019 (COVID-19) - United States, February 12March 16, 2020. MMWR Morb Mortal Wkly Rep 2020;69(12):343-46. doi: https://doi.org/10.15585/mmwr.mm6912e2 [published Online First: 2020/03/28]

2. Centers for Disease Control and Prevention. Provisional death counts for coronavirus disease (COVID-19) [Available from: https://www.cdc.gov/ nchs/nvss/vsrr/covid19/index.htm accessed 2020/06/11

3. New York City Department of Health and Mental Hygeine. COVID-19: data [Available from: https://www1.nyc.gov/site/doh/covid/covid-19-data. page accessed 2020/06/11.

4. Brown EE, Kumar S, Rajii TK, et al. Anticipating and mitigating the impact of the COVID-19 pandemic on Alzheimer's disease and related dementias. Am J Geriatr Psychiatry 2020 doi: https://doi.org/10.1016/j. jagp.2020.04.010 [published Online First: 2020/04/26]

5. Centers for Medicare \& Medicaid Services. Physicians and other clinicians: CMS flexibilities to fight COVID-19 [Available from: https://www.cms.gov/ files/document/covid-19-physicians-and-practitioners.pdf accessed 2020/6/11.

6. Centers for Medicare \& Medicaid Services. CMS takes action nationwide to aggressively respond to coronavirus national emergency [Available from: https://www.cms.gov/newsroom/press-releases/cms-takes-action-nationwide-aggressively-respond-coronavirus-national-emergency accessed 2020/06/11.

7. Lagu T. The importance of compassion as the default in times of crisis: the curious case of "vertical interdiction". J Hosp Med 2020; 15(5):316-17. doi: https://doi.org/10.12788/jhm.3428 [published Online First: 2020/05/ 08]

Publisher's note Springer Nature remains neutral with regard to jurisdictional claims in published maps and institutional affiliations. 